\title{
Treatment for pulmonary manifestations of juvenile systemic sclerosis
}

\author{
Gretchen Henstorf $^{1 *}$, Anne M Stevens ${ }^{2}$ \\ From 2011 Pediatric Rheumatology Symposium sponsored by the American College of Rheumatology \\ Miami, FL, USA. 2-5 June 2011
}

\begin{abstract}
Purpose
Children with systemic sclerosis (SSc) may develop severe alveolitis with progressive, potentially fatal interstitial fibrosis. There have been no controlled trials for treatment of juvenile SSc. Adult trials suggest benefit from cyclophosphamide (CY) therapy, though with significant risk for adverse events. As part of a larger study to develop objective clinical and radiological measures for outcomes in juvenile SSc, we reviewed the courses of seven patients with SSc lung disease who were treated with extensive courses of $\mathrm{CY}$ or methotrexate.
\end{abstract}

\section{Methods}

Medical records were reviewed for all patients diagnosed with scleroderma at Seattle Children's Hospital during the years 1994 to 2010 to identify children who fulfilled ACR criteria for systemic sclerosis. Nineteen patients were identified: 15 girls and four boys. Eleven patients developed pulmonary fibrosis and/or alveolitis detected by high resolution CT scan. Further review was conducted of records for seven patients with pulmonary SSc followed for a mean of 6.4 years (range 4.1-9.6). The mean age at diagnosis was 10.8 years (range 6-14.7).

\section{Results}

The DLCO adjusted for alveolar volume (DLCO) prior to therapy averaged $81.9 \%$, ranging from $68-99 \%$ of normal. Five patients (mean DLCO 79.6\%, range 68-99\%) were treated with $\mathrm{CY}$ at the first sign of alveolitis. The $\mathrm{CY}$ treatment regimen included oral CY (1-3 $\mathrm{mg} / \mathrm{kg} /$ day $)$ and/or IV CY ( $750 \mathrm{mg} / \mathrm{m} 2 / \mathrm{month}$ ) for an average of 1.6 years (range 0.5 to 3 ). The cumulative $\mathrm{CY}$ doses averaged 19 grams (range 7 to 28.1 grams). Two patients (DLCO $81 \%$ and $94 \%$ ) were treated with methotrexate $1 \mathrm{mg} / \mathrm{kg}$, with a maxiumum $40 \mathrm{mg}$, subcutaneously once a week. Corticosteroids were

${ }^{1}$ Seattle Children's Research Institute, Seattle, WA, USA

Full list of author information is available at the end of the article administered to all patients. We found that three of the five $\mathrm{CY}$ patients had improved pulmonary function at the last test, with a mean decrease in DLCO of $2.2 \%$ (range -14 to $+5 \%$, mean $-0.43 \% /$ year). Patients on methotrexate were stable with DLCO improving in one (81 to $102 \%$ ) and decreasing in one (94\% to $81 \%$ ). Serial CT scans showed stable or improved disease (by estimated area of alveolitis and/or fibrosis) in all seven patients. During or after CY therapy there was one hospitalization for possible serious infection, eventually attributed to antibiotic-induced pneumonitis, and one case of otitis media. One patient had mild gastritis that resolved with a reduction in oral CY dose. Pulmonary hypertension was detected and resolved in three patients. No malignancies developed.

\section{Conclusion}

Juvenile SSc patients with severe SSc lung disease can stabilize and even improve with cyclophosphamide or methotrexate and corticosteroid therapy. The low shortterm rate of adverse events reported here and high rate of morbidity and mortality of SSc without treatment argues for the benefit of cyclophosphamide or methotrexate in children with SSc lung disease.

\section{Disclosure}

Gretchen Henstorf: None; Anne M. Stevens: None.

\section{Author details}

${ }^{1}$ Seattle Children's Research Institute, Seattle, WA, USA. ' University of Washington, Seattle, WA, USA.

Published: 13 July 2012

doi:10.1186/1546-0096-10-S1-A70

Cite this article as: Henstorf and Stevens: Treatment for pulmonary manifestations of juvenile systemic sclerosis. Pediatric Rheumatology 2012 10(Suppl 1):A70.

\section{Biomed Central}

(c) 2012 Henstorf and Stevens; licensee BioMed Central Ltd. This is an Open Access article distributed under the terms of the Creative Commons Attribution License (http://creativecommons.org/licenses/by/2.0), which permits unrestricted use, distribution, and reproduction in any medium, provided the original work is properly cited. 